\title{
La pandemia de la COVID-19 en el Perú: análisis epidemiológico de la segunda ola
}

\section{The COVID-19 pandemic in Peru: epidemiological analysis of the second wave}

\author{
James Walter Gutiérrez-Tudelal
}

Luego de la declinación de la primera ola en el mes de diciembre del año 2020, pero sin llegar a un porcentaje de casos acumulados de la Covid-19 del 5\% (este valor representa el porcentaje de casos positivos en relación al total de pruebas acumuladas realizadas) ya que este todavía se mantuvo por encima del 18\%, los casos diarios empezaron a elevarse la segunda quincena del mes de diciembre, haciendo que los contagios en promedio semanales fueron mayores que el promedio de los dados de alta semanales. ${ }^{1}$ Igualmente, el porcentaje de crecimiento que durante el mes de diciembre había bajado a $0,14 \%$ en promedio semanal y luego empezó a aumentar, pero sin llegar en esta oportunidad siquiera al $1 \%$ en promedio semanal (cifra que representa los contagios de un día en comparación con los contagios acumuladas hasta el día anterior) en comparación con más del $20 \%$ en promedio semanal que se había alcanzado durante las primeras semanas de la primera ola el año 2020. Por otro lado, el porcentaje de crecimiento en la segunda ola alcanzó un máximo de $0,60 \%$ en promedio semanal en la semana 54 , ésta contada después el inicio de la pandemia en nuestro país, es decir la cuarta semana del mes de marzo del año 2021. Posteriormente, este porcentaje de crecimiento fue disminuyendo por debajo del $0,10 \%$ en promedio semanal hasta fines de octubre del año 2021.

Internista e infectólogo, FACP. Presidente de la Sociedad Peruana de Medicina Interna, Lima, Perú.
Gutiérrez-Tudela JW. La pandemia de la COVID-19 en el Perú: análisis epidemiológico de la segunda ola. Rev Soc Peru Med Interna. 202 I;34(4):I29-I30. DOI: https://doi.org/10.36393/spmi.v34i4.627

Luego durante el mes de enero del año 2021 hubo un aumento sostenido de los casos o contagios, haciendo que estos fueran en promedio más elevados que las altas también en promedio. Así durante la semana 43, entre el 04 y el 10 de enero de este año, los contagios en promedio semanal eran de 2,244 casos diarios y las altas solamente 1, 597 casos diarios, también en promedio. El promedio o media semanal se calculó sumando los casos durante siete días y dividiendo el resultado entre $7 .^{2} \mathrm{El}$ mayor promedio semanal se alcanzó durante la semana 54, entre el 22 y el 28 de marzo, con 9,079 contagios diarios y 8,727 altas diarias en promedio semanal. A partir de la semana 67 , entre el 21 y el 27 de Junio, en promedio las altas fueron mayores que los contagios, tendencia que persistió hasta fines de octubre de este año 2021, es decir durante los meses de julio, agosto, setiembre y octubre, hasta la semana 85 post inicio de la pandemia en nuestra patria, siendo la semana del 25 al 31 de octubre en que hubo un promedio diario de 847 contagios y 926 altas.

Es importante mencionar también que, durante la segunda quincena del mes de diciembre del año 2020, los contagios acumulados superaron el millón de casos de la infección por el SARS-Cov-2 (1 millón 153 mil casos, el 22 de diciembre) desde que se reportó el primer paciente en el mes de marzo de este año. Sin embargo, ya se advertía desde hace meses atrás un subregistro de miles de pacientes infectados por las deficiencias en el sistema de notificación, habiendo un desfase entre las cifras oficiales que reportaba el Ministerio de Salud (MINSA) y las Direcciones Regionales de Salud (DIRESA's), al extremo que estos últimos calculaban el doble de los casos totales reportados por el MINSA.

Teóricamente y haciendo un ejercicio, se pudo inferir 
en vista de que el porcentaje de positividad acumulada en ese momento era del $18,5 \%$ (esta cifra en relación al número total de pruebas acumuladas) y extrapolando a la población en general del Perú de 32'658, 512 habitantes, que el número de casos o contagios acumulados con la infección por el nuevo coronavirus podría haber superado los 6 millones de casos de pacientes infectados, es decir casi el triple de casos totales reportados por las DIRESAS. Este cálculo, vuelvo a repetir, es teórico y no representa la realidad de los hechos.

Durante la segunda quincena del mes de marzo del año 2021 y casi durante todo el mes de abril de este año se tuvo el mayor número de casos o contagios diarios, siendo el día 15 de abril el de mayor valor con 13326 contagios, seguido por el día 1 de abril con 12916 casos y el día 26 de marzo con 11919 contagios o casos diagnosticados, cifras que no se habían encontrado durante la primera ola de la actual pandemia. Los casos positivos de la COVID-19 durante las primeras semanas de la segunda ola, según etapas de la vida, fueron mayormente en los adultos (entre 30 y 59 años) con una tasa de ataque de 6,96 y luego los adultos mayores (de 60 años a más) con una tasa de ataque de 6,56. La tasa de ataque se refiere al número de casos por 100 habitantes. ${ }^{3}$ Así, el riesgo de enfermarse fue de $49 \%$ para los adultos y de $40 \%$ para los adultos mayores. El 51,6\% correspondió al sexo masculino y el $48,4 \%$ al sexo femenino. Tomando en cuenta el total de casos acumulados, el mayor porcentaje correspondía a los adultos, con el 57\%, luego los jóvenes (de 18 a 29 años) con un 19\%. En tercer lugar, los adolescentes (de 12 a 17 años) con un 3\% y, finalmente, los niños (de 0 a 11 años) también con un 3\%, grupo etario este último en el que hubo un ligero aumento de casos.

La tasa de ataque acumulada por macrorregiones al comienzo de la segunda ola fue en este orden: primero Lima y Callao, luego el oriente, seguido por el sur, el centro y el norte. La tasa de ataque acumulada por regiones fue predominantemente para los departamentos fronterizos y selváticos con menor población. Así Moquegua estuvo en primer lugar con 8,59; luego, Madre de Dios con 5,54; Amazonas, con 4,39; Lima, con 4,36; Tacna, con 4,07; Callao, con 3,95; Tumbes, con 3,84 ; Ucayali con 3,51 ; Ica con 3,36; Arequipa con 3,31; y, el promedio para el Perú fue de 3,20. En el pico más alto de la segunda ola, entre los meses de marzo y abril del año 2021, las regiones con mayores tasas de ataque acumulada fueron los mismos, pero en este orden: Moquegua con el 11,21; Lima, 7,47; Madre de Dios 6,62; Callao 6,21; Tacna 5,89; Amazonas 5,40; Tumbes 5,29; Ica 4,54; Ucayali 4,46; Arequipa 4,35; $\mathrm{y}$, el promedio nacional 4,84 .

En cuanto a la mortalidad, recién el día 31 de mayo del presente año, el Sistema Nacional de Defunciones (SINADEF) del MINSA sinceró las cifras que se suponía eran las reales, pasando de 69342 decesos totales a la cifra de 184507 fallecidos el día uno de junio del 2021, representando esta cifra más de dos veces y media de las cifras que se estuvieron dando oficialmente en forma diaria desde el año pasado. La tasa de letalidad igualmente pasó de $3,54 \%$ a la cifra de $9,40 \%$, la misma que representaba entre 9 a 10 muertos por cada 100 pacientes infectados con la SARS-CoV-2. La tasa de letalidad se refiere al número de fallecidos por cada 100 infectados o enfermos. ${ }^{4}$ Estas cifras representaban una de las tasas de mortalidad más altas en el mundo por 100000 habitantes y el Perú se ubicaba en ese momento en el quinto país en el mundo con mayor número de defunciones después de Estados Unidos de Norteamérica, Brasil, India y México, países que tienen centenares de millones de habitantes en comparación con el nuestro. Este hecho se debe al mal manejo que se hizo de la pandemia de la COVID-19 desde su aparición en nuestro país en el mes de marzo del 2020 en relación a las medidas que se tomaron y a las que debían haberse tomado para contener y mitigar la pandemia. La cifra de fallecidos totales a fines del mes de octubre del presente año era de 200 276, representando una tasa de letalidad del 9,1\% o sea 9 fallecidos por cada 100 pacientes infectados.

\section{REFERENCIAS BIBLIOGRÁFICAS}

I. Sala situacional. Centro Nacional de Epidemiología y Control de Enfermedades. MINSA, Perú; 2020.

2. Beaglehole R, Bonita R \& Kjellstrom T. Basic Epidemiology, 2nd edition. PAHO,WHO. 2008

3. Norman GR and Streiner DL. Biostatistics. The Bare Essentials. 4th Edition. People's Medical Publishing House. 2014.

4. Petrie A and Sabin C. Medical Statistics at a Glance. Fourth edition. Wiley Blackwell. 2020. 